Supplement of Atmos. Chem. Phys. Discuss., 15, 14007-14026, 2015

http://www.atmos-chem-phys-discuss.net/15/14007/2015/

doi:10.5194/acpd-15-14007-2015-supplement

(C) Author(s) 2015. CC Attribution 3.0 License.

(c) (i)

\title{
A study of the impact of synoptic weather conditions and water vapor on aerosol-cloud relationships over major urban clusters of China
}

K. Kourtidis et al.

Correspondence to: K. Kourtidis (kourtidi@env.duth.gr)

The copyright of individual parts of the supplement might differ from the CC-BY 3.0 licence. 

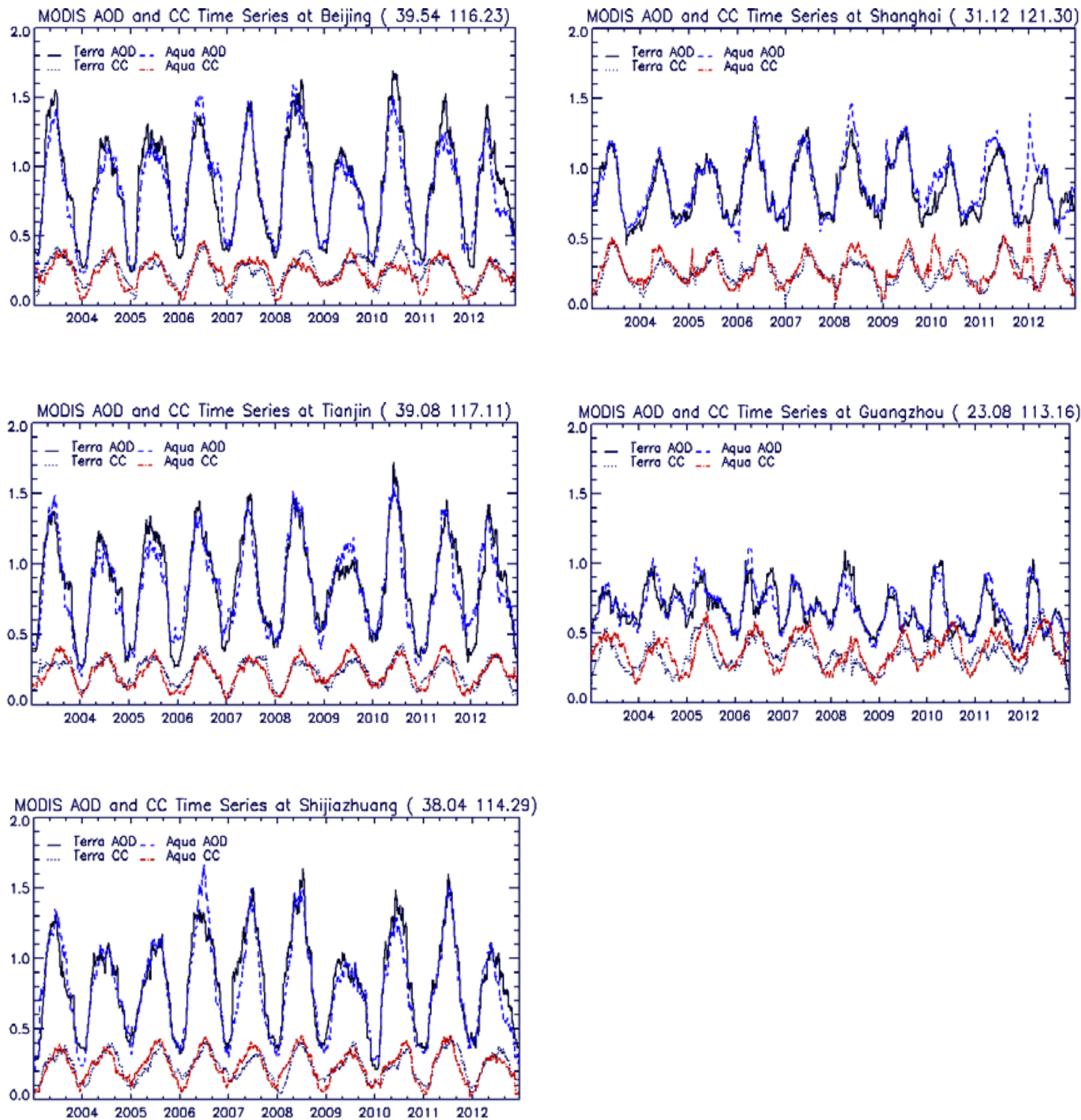

Figure S1. AOD and CC timeseries from TERRA and AQUA satellites at Beijing (upper left), Shanghai (upper right), Tianjin (middle left), Guangzhou (middle right) and Shijiazhuang (lower left), for the time period 2003 - 2013. The three cities on the left are within the BTH cluster, Shanghai lies within the YRD cluster and Guangzhou lies within the PRD cluster. 

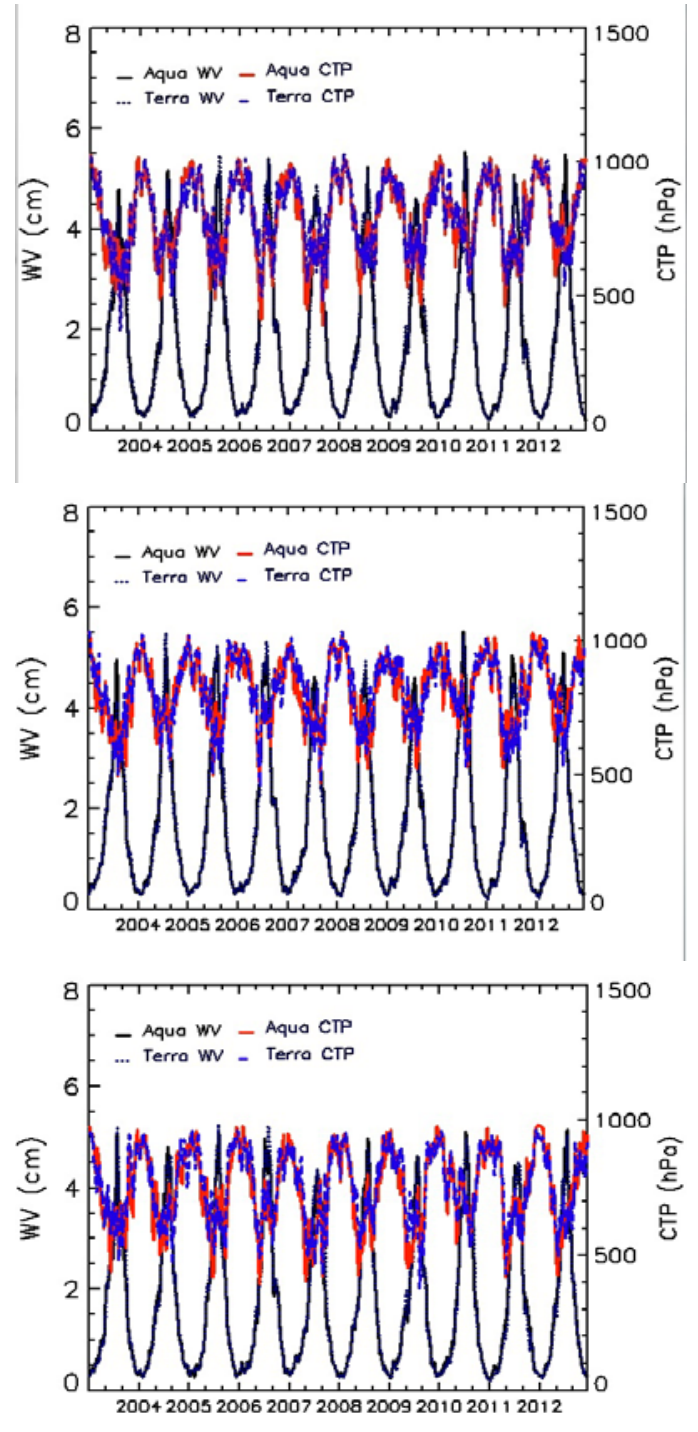
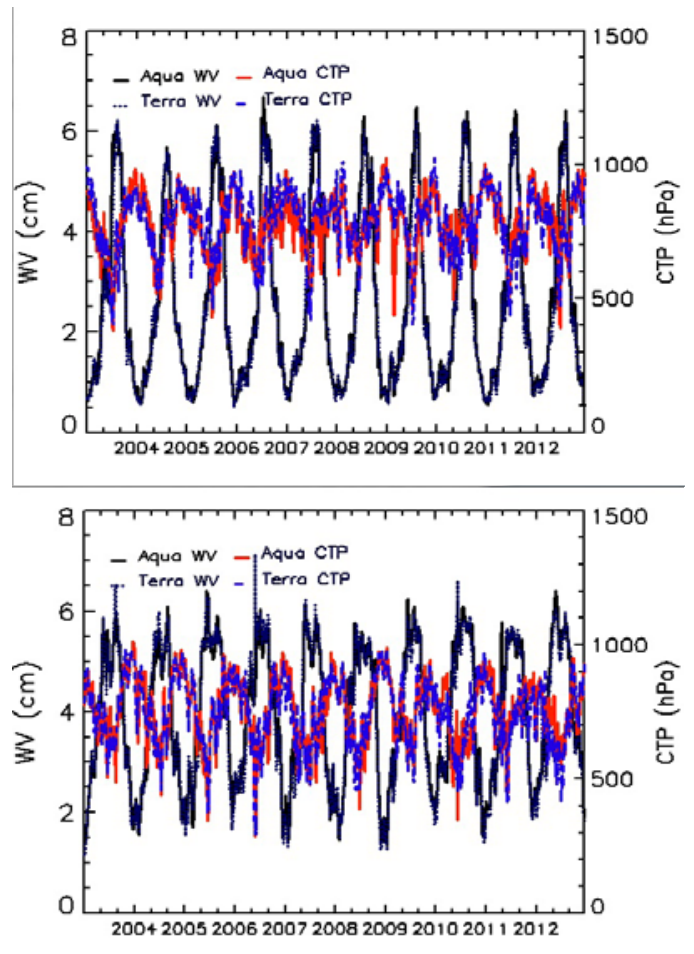

Figure S2. WV and CTP timeseries from TERRA and AQUA satellites at Beijing (upper left), Shanghai (upper right), Tianjin (middle left), Guangzhou (middle right) and Shijiazhuang (lower left), for the time period 2003-2013. The three cities on the left are within the BTH cluster, Shanghai lies within the YRD cluster and Guangzhou lies within the PRD cluster. 
a)

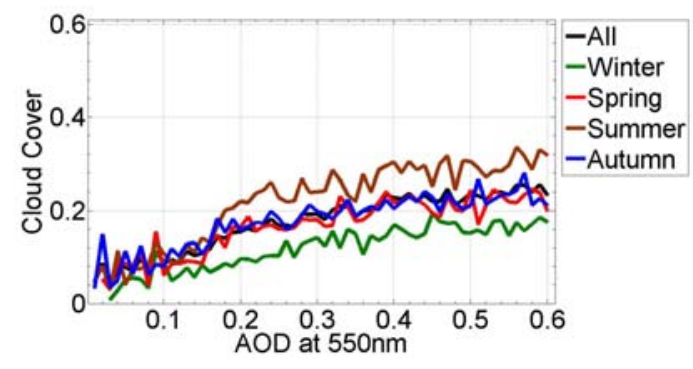

b)

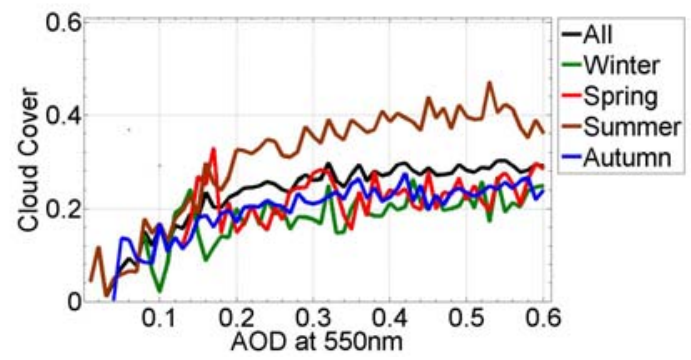

c)

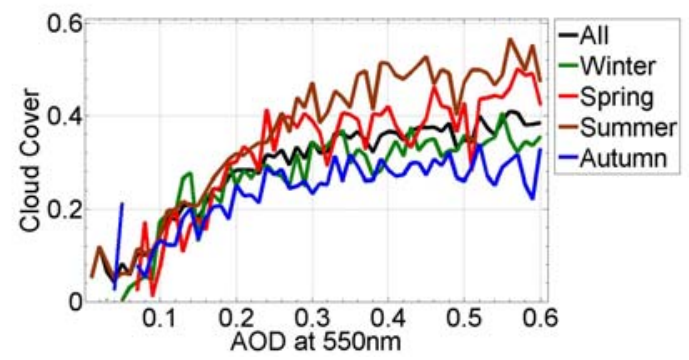

d)

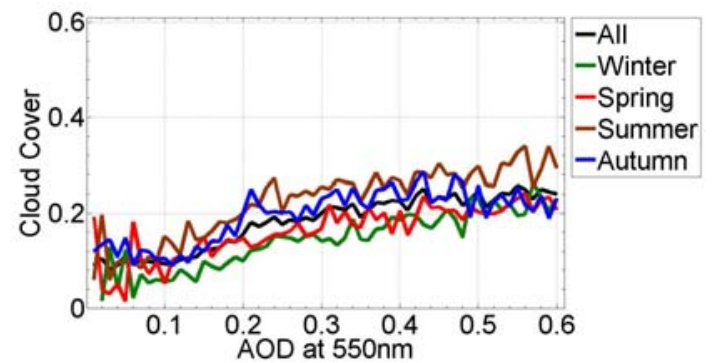

e)

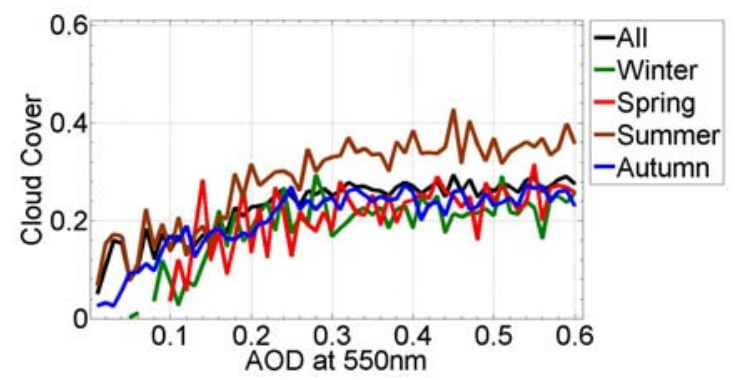

f)

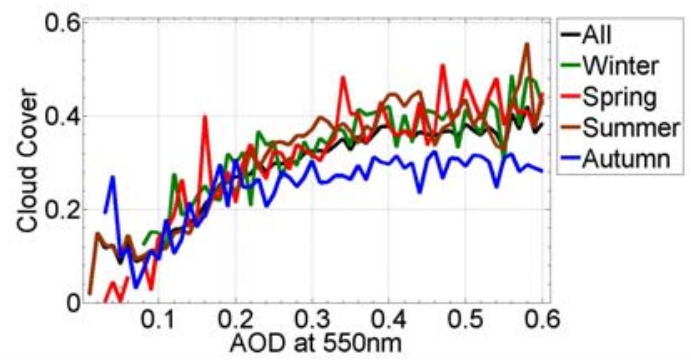

Figure S3. Annual and seasonal AOD as a function of CC over BTH $(\mathbf{a}, \mathbf{d}), \operatorname{YRD}(\mathbf{b}, \mathbf{e})$ and PRD $(\mathbf{c}, \mathbf{f})$ from AQUA (a-c) and TERRA (d-f) satellites for the time period 2003-2013. 
Table S1. AOD-CC slopes for 0-0.22 AOD over BTH, YRD and PRD from AQUA and TERRA satellites for the time period 2003-2013. The values in parentheses represent $R^{2}$ coefficients. The asterisks denote statistically significant values at the $95 \%$ confidence level $(a=0.05)$.

\begin{tabular}{cccccc}
\hline \multicolumn{7}{c}{ Aqua } \\
\hline & All Seasons & Winter & Spring & Summer & Autumn \\
\hline BTH & $0.51(0.83)^{*}$ & $0.31(0.50)^{*}$ & $0.55(0.60)^{*}$ & $0.85(0.83)^{*}$ & $0.56(0.61)^{*}$ \\
YRD & $1.02(0.87)^{*}$ & $-0.06(0.001)$ & $-0.52(0.08)$ & $1.43(0.88)^{*}$ & $0.75(0.65)^{*}$ \\
PRD & $1.21(0.92)^{*}$ & $1.62(0.78)^{*}$ & $1.87(0.82)^{*}$ & $1.42(0.93)^{*}$ & $0.89(0.58)^{*}$ \\
\hline \multicolumn{7}{c}{ Terra } \\
\hline BTH & $0.36(0.73)^{*}$ & $0.28(0.28)^{*}$ & $0.33(0.21)^{*}$ & $0.60(0.63)^{*}$ & $0.40(0.39)^{*}$ \\
YRD & $0.64(0.70)^{*}$ & $1.34(0.81)^{*}$ & $0.76(0.16)$ & $0.80(0.60)^{*}$ & $0.77(0.80)^{*}$ \\
PRD & $1.00(0.82)^{*}$ & $1.30(0.67)^{*}$ & $1.62(0.77)^{*}$ & $0.98(0.77)^{*}$ & $0.78(0.35)^{*}$ \\
\hline
\end{tabular}

Table S2. AOD-CC slopes for 0.23-0.6 AOD over BTH, YRD and PRD from AQUA and TERRA satellites for the time period 2003-2013. The values in parentheses represent $R^{2}$ coefficients. The asterisks denote statistically significant values at the $95 \%$ confidence level $(a=0.05)$.

\begin{tabular}{cccccc}
\hline \multicolumn{7}{c}{ Aqua } \\
\hline & All Seasons & Winter & Spring & Summer & Autumn \\
\hline BTH & $0.21(0.81)^{*}$ & $0.17(0.59)^{*}$ & $0.17(0.48)^{*}$ & $0.22(0.55)^{*}$ & $0.16(0.50)^{*}$ \\
YRD & $0.11(0.50)^{*}$ & $0.15(0.27)^{*}$ & $0.13(0.14)^{*}$ & $0.19(0.38)^{*}$ & $0.09(0.27)^{*}$ \\
PRD & $0.22(0.64)^{*}$ & $0.18(0.35)^{*}$ & $0.28(0.33)^{*}$ & $0.38(0.56)^{*}$ & $0.09(0.11)^{*}$ \\
\hline \multicolumn{7}{c}{ Terra } \\
\hline BTH & $0.18(0.74)^{*}$ & $0.26(0.71)^{*}$ & $0.18(0.56)^{*}$ & $0.19(0.48)^{*}$ & $0.04(0.03)$ \\
YRD & $0.06(0.27)^{*}$ & $0.06(0.05)$ & $0.15(0.20)^{*}$ & $0.15(0.28)^{*}$ & $0.03(0.03)$ \\
PRD & $0.23(0.68)^{*}$ & $0.29(0.40)^{*}$ & $0.36(0.46)^{*}$ & $0.24(0.27)^{*}$ & $0.13(0.27)^{*}$ \\
\hline
\end{tabular}

Table S3. AOD-CC and AOD-CTP slopes for different WV bins from Fig. 2 over the BTH region for SLP $<1008$ and SLP $>1017$ from AQUA for the time period 2003-2013. The values in parentheses represent $R^{2}$ coefficients. The asterisks denote statistically significant values at the $95 \%$ confidence level $(a=0.05) .(\sim)$ denotes very small value.

\begin{tabular}{ccccc}
\hline & \multicolumn{2}{c}{ SLP $<1008$} & \multicolumn{2}{c}{ SLP > 1017 } \\
\hline $\begin{array}{c}\text { WV } \\
\text { class }\end{array}$ & AOD-CC & AOD-CTP & AOD-CC & AOD-CTP \\
\hline $\mathbf{1} \mathbf{~ c m}$ & $0.15(0.57)^{*}$ & $151.20(0.65)^{*}$ & $0.19(0.93)^{*}$ & $-7.67(0.01)$ \\
$\mathbf{2} \mathbf{~ c m}$ & $0.19(0.89)^{*}$ & $28.36(0.13)$ & $0.07(0.57)^{*}$ & $180.50(0.81)^{*}$ \\
$\mathbf{3} \mathbf{~ c m}$ & $0.15(0.76)^{*}$ & $35.97(0.12)$ & $0.01(\sim)$ & $132.30(0.65)^{*}$ \\
$\mathbf{4} \mathbf{c m}$ & $0.15(0.47)^{*}$ & $-38.72(0.17)$ & $0.27(0.28)$ & $-295.10(0.72)$ \\
$\mathbf{5} \mathbf{~ c m}$ & $0.11(0.64)^{*}$ & $-23.01(0.05)$ & - & - \\
$\mathbf{6} \mathbf{c m}$ & $0.14(0.25)$ & $29.47(0.02)$ & - & - \\
$\mathbf{7} \mathbf{~ c m}$ & $0.28(0.65)^{*}$ & $-151.90(0.43)$ & - & - \\
$\mathbf{8} \mathbf{~ c m}$ & $0.38(0.23)$ & $-181.9(0.83)$ & - & - \\
$\mathbf{9} \mathbf{~ c m}$ & - & - & - & - \\
$\mathbf{1 0} \mathbf{~ c m}$ & - & - & - & - \\
\hline
\end{tabular}


Table S4. AOD-CC and AOD-CTP slopes for different WV bins from Fig. 3 over the YRD region for SLP $<1008$ and SLP > 1017 from AQUA for the time period 2003-2013. The values in parentheses represent $R^{2}$ coefficients. The asterisks denote statistically significant values at the $95 \%$ confidence level $(a=0.05) .(\sim)$ denotes very small value.

${ }^{1}:$ This value represents a line of 2 points

\begin{tabular}{ccccc}
\hline & \multicolumn{2}{c}{ SLP $<1008$} & \multicolumn{2}{c}{ SLP > 1017 } \\
\hline $\begin{array}{c}\mathbf{W V} \\
\text { class }\end{array}$ & AOD-CC & AOD-CTP & AOD-CC & AOD-CTP \\
\hline $\mathbf{1} \mathbf{~ c m}$ & - & - & $0.07(0.71)^{*}$ & $219.30(0.48)^{*}$ \\
$\mathbf{2} \mathbf{~ c m}$ & $0.43(0.67)^{*}$ & $-197.40(0.22)$ & $0.21(0.82)^{*}$ & $-22.55(0.02)$ \\
$\mathbf{3} \mathbf{~ c m}$ & $0.05(0.14)$ & $161.60(0.92)^{*}$ & $0.18(0.88)^{*}$ & $44.28(0.45)^{*}$ \\
$\mathbf{4} \mathbf{~ c m}$ & $0.20(0.67)^{*}$ & $-82.04(0.19)$ & $0.33(0.84)^{*}$ & $-5.73(\sim)$ \\
$\mathbf{5} \mathbf{~ c m}$ & $0.28(0.75)^{*}$ & $-132.10(0.63)^{*}$ & $0.14(0.28)$ & $-7.55(\sim)$ \\
$\mathbf{6} \mathbf{c m}$ & $0.25(0.76)^{*}$ & $-207.10(0.84)^{*}$ & $-0.66(1)^{1}$ & - \\
$\mathbf{7} \mathbf{~ c m}$ & $0.11(0.57)^{*}$ & $-140.60(0.78)^{*}$ & - & - \\
$\mathbf{8} \mathbf{~ c m}$ & $0.18(0.42)$ & $-20.77(0.02)$ & - & - \\
$\mathbf{9} \mathbf{~ c m}$ & - & - & - & - \\
$\mathbf{1 0} \mathbf{~ c m}$ & - & - & - & - \\
\hline
\end{tabular}

Table S5. AOD-CC and AOD-CTP slopes for different WV bins from Fig. 4 over the PRD region for SLP $<1008$ and SLP > 1017 from AQUA for the time period 2003-2013. The values in parentheses represent $R^{2}$ coefficients. The asterisks denote statistically significant values at the $95 \%$ confidence level $(a=0.05) .(\sim)$ denotes very small value.

\begin{tabular}{ccccc}
\hline & \multicolumn{2}{c}{ SLP $<1008$} & \multicolumn{2}{c}{ SLP > 1017 } \\
\hline $\begin{array}{c}\mathbf{W V} \\
\text { class }\end{array}$ & AOD-CC & AOD-CTP & AOD-CC & AOD-CTP \\
\hline $\mathbf{1} \mathbf{~ c m}$ & - & - & $0.01(\sim)$ & $915.00(0.70)$ \\
$\mathbf{2} \mathbf{~ c m}$ & - & - & $0.03(0.03)$ & $2.64(\sim)$ \\
$\mathbf{3} \mathbf{~ c m}$ & - & - & $0.07(0.29)$ & $36.62(0.23)$ \\
$\mathbf{4} \mathbf{~ c m}$ & $-0.13(0.21)$ & $-38.09(0.02)$ & $0.08(0.32)$ & $85.05(0.66)^{*}$ \\
$\mathbf{5} \mathbf{~ c m}$ & $0.18(0.34)$ & $-242.70(0.71)^{*}$ & $0.07(0.06)$ & $43.99(0.19)$ \\
$\mathbf{6} \mathbf{~ c m}$ & $0.17(0.51)^{*}$ & $-175.90(0.87)^{*}$ & - & - \\
$\mathbf{7} \mathbf{~ c m}$ & $0.14(0.32)$ & $-165.90(0.42)^{*}$ & - & - \\
$\mathbf{8} \mathbf{~ c m}$ & - & - & - & - \\
$\mathbf{9} \mathbf{~ c m}$ & - & - & - & - \\
$\mathbf{1 0} \mathbf{~ c m}$ & - & - & - & - \\
\hline
\end{tabular}

